\title{
Usability Testing of Mobile Augmented Reality Applications for Cultural Heritage - A Systematic Literature Review
}

\author{
Diana Tiriteu, Silviu Vert \\ Communications Department, Politehnica University of Timişoara \\ Piata Victoriei No. 2, Timişoara, Romania \\ diana.tiriteu@student.upt.ro, silviu.vert@upt.ro
}

DOI: $10.37789 /$ rochi.2020.1.1.21

\begin{abstract}
This paper presents an overview of the existing literature to date regarding the usability testing of Mobile Augmented Reality applications developed for cultural heritage. A Systematic Literature Review was conducted with this purpose. Four databases were interrogated with specific keywords. Our of the 88 found research papers, only 31 met the eligibility criteria to be included in this survey. Four major usability testing methods were identified: questionnaires, focus groups, user testing and interviews. The questionnaire method was used in almost all of the studies, and only a few of them combined more usability testing methods. Nearly all papers targeted the outdoors use centered on location-based augmented reality. The perceived ease of use, usefulness and enjoyment were confirmed by a majority of the papers. The ease of use was also correlated to the ease of learning, as well as with the interest of using the application. Cultural heritage is one of the domains that can benefit greatly from the latest improvements in mobile augmented reality technologies.
\end{abstract}

\section{Author Keywords}

Mobile augmented reality; usability; user experience; questionnaire; focus group; user testing; interview; cultural heritage.

\section{ACM Classification Keywords}

- General and reference Document types Surveys and overviews - Human-centered computing Human computer interaction $(\mathrm{HCI}) \sim$ Interaction paradigms $\sim$ Mixed / augmented reality $\bullet$ Human-centered computing $\sim$ Human computer interaction $(\mathrm{HCI}) \sim \mathrm{HCI}$ design and evaluation methods $\sim$ Usability testing

\section{INTRODUCTION}

This paper focuses on the usability testing of Mobile Augmented Reality (MAR) when applied for cultural heritage. In the past few years, cutting-edge technology has developed tremendously in smartphones, making it more approachable for all people. Along with this, application developers took advantage of this new fast-growing applicable domain and started creating apps for almost everything. Amongst those, Augmented Reality (AR) has grown in the mobile applications area, having now the widest audience ever. Together with application developers, cultural institutions are benefiting from this technology growth by trying to design and implement applications which support the cultural heritage [3]. And what better technology to fit their needs than AR? However, just having an app that seems to fit your need does not mean that the people using it are having the best user experience possible. Here, an important aspect is represented by the usability of the app.

User Experience ( $U X)$ covers all aspects of the end-user's interaction with an application. In a proposed model by Lee [23], UX is a combination of usability (perceived usefulness, ease of use and enjoyment) and personal characteristics. Considering this, the usability of an application will be high if the users will find the app easy to use, efficient and enjoyable.

\begin{abstract}
Augmented Reality (AR)
Augmented Reality is a technology used to enhance the context of the real world. It works by overlaying digital representations on the objects from the physical world. Doing so, it enriches the user's perceptions on seeing, hearing, and feeling. All of this can be accomplished with the camera lens of a smartphone or tablet. A good AR design makes the virtual and the physical world coexist harmoniously, the user getting to perceive the overlaid AR elements as being part of the physical world. There are four different categories of AR technologies which are well described by Vanessa Camilleri in [36], and all of them are adaptable to different contexts:
\end{abstract}

Marker-based AR uses virtual markers that, when sensed by the reader (e.g. the mobile phone's camera), trigger a result. This type of AR uses the image recognition technology, and one of the simplest markers can be found in QR codes.

Markerless AR uses location-based technologies or GPS to provide data based on the location of the mobile device. The accelerometer embedded in smartphones provides the location coordinates for the app and activates the AR data. 
This type of AR is mostly used in tourism and marketing apps, as it makes use of the exact location and provides information about the nearby attractions or places of interest.

Projection-based AR uses projected light by combining cameras to 3D sensing systems (e.g. depth cameras) and allowing digital displays to appear on any surface. This type of AR has applications in operations, manufacturing, and other fields. A popular example may be represented by the projected images on buildings, usually at Christmas markets at night.

The last type of AR is the superimposition-based AR. This one replaces partially or wholly an existing object from the real world with an augmented view of it. A famous example of this technology being used is an application from IKEA, with which customers can superimpose furniture from the online retailer right in their homes, to see how it would look like. Image recognition plays a vital role here, otherwise there is nothing to be replaced.

$\mathrm{AR}$ has uses in entertainment, education, art, tourism, and cultural heritage, as well as others. Today, the number of smartphone users is around 3.5 billion [39], making Mobile AR (MAR) more accessible than ever via social media apps, gaming and others.

\section{Cultural Heritage}

According to Bill Ivey, Cultural Heritage (CH) "tells us where we came from by preserving and presenting voices from the past, grounding us in the linkages of family, community, ethnicity, and nationality, giving us our creative vocabulary" [35]. Cultural heritage can also be split in tangible and intangible. Tangible $\mathrm{CH}$ refers to physical artifacts, including artistic creations and other tangible products that have a cultural importance. Intangible $\mathrm{CH}$ refers to non-physical practices, expressions, knowledge, artifacts and cultural spaces associated with communities, groups and individuals [2]. Due to the paramount importance of heritage, cultural institutions are safeguarding the world's art, culture, history and heritage for hundreds of years already. With the new technologies of today and tomorrow, this safeguarding mission may become easier, with an even wider audience - a worldwide one. When the access to the art and heritage of people's past is restricted, understanding human nature becomes more difficult. Digital technologies and web-based communication platforms remove the obstacles in disseminating knowledge and cultural heritage to people.

Being already an advanced technology, AR is a great way to meet the needs of cultural heritage. Using AR in the cultural heritage context maximizes visitor's satisfaction and offers a unique, personalized experience to each tourist [29]. If the user experience and the usability of the application are also taken into consideration, it is even more accessible for the cultural heritage to reach its goal of being noticed.

In this paper the taxonomy for culture is empirically linked to cultural heritage, tourism, art, and museums, based on the surveyed papers and on the lack of a standard taxonomy for this domain.

\section{RELATED WORK}

As AR started to develop in the cultural heritage field, there are already literature reviews which aim to present the trends in this domain.

Aliprantis et al. [3] surveyed in 2019 the trends in building AR applications for the cultural heritage field. They divided the different AR approaches into 8 categories: serious games, personalization, AR reconstruction, projection display, Semantics/Linked Open Data, cultural UX evaluation, Context Awareness and digital storytelling. Considering the amount of the various approaches, the authors assume that the evaluation of the Cultural UX is one of the most active fields in AR applications for cultural heritage, as institutions constantly try to adapt to their visitor's needs. Most of the papers analyzed in this survey used the simplest evaluation methods, as a short questionnaire, in order to obtain feedback from the visitors. Another active field in AR applications for cultural heritage is digital reconstruction. Recent works in the cultural heritage field present multiple and different techniques to virtually reconstruct cultural relics or monuments and provide an immersive experience to their users.

Bekele et al. [4] surveyed augmented, virtual, and mixed reality from a cultural heritage perspective. On the AR topic, the authors identified that AR applications in the cultural heritage domain frequently use marked-based, markerless, and hybrid tracking approaches. In a hybrid approach, the camera of the device and electromagnetic, inertial, and acoustic sensors are used. They categorize AR systems in two types: indoor and outdoor AR. Indoor AR uses markerbased tracking, and usually does not require the use of GPS. Outdoor AR relies heavily on markerless and hybrid tracking. They also classified the purpose of augmented, virtual and mixed reality when used for cultural heritage:

- Education aims at enabling users to learn the historical aspects.

- Exhibition enhancement at physical museums and heritage sites.

- Exploration supports users in visualizing and exploring historical and current views of cultural heritage.

- Reconstruction enables users to picture and interact with reconstructed historical views of cultural heritage.

- Virtual museums simulate and present cultural heritage (both tangible and intangible) to the public.

The survey showed that AR is preferable for exhibition enhancement.

Another survey presented the cultural heritage in markerless AR [21]. The authors classify the AR techniques in two main categories: vision-based AR and location-based AR. The study summarizes the existing research on markerless AR, 
both for indoor and outdoor use. It also identifies four main issues related to AR when used for cultural heritage. The first concern is related to registration and it refers to the compatibility of augmented objects with the real environment. The second topic is reconstruction, which is a construction of virtual objects in a way to replicate the original building - this is usually done by using 3D scanning techniques. The third issue refers to tracking, where a high level of accuracy and a low level of latency are the key requirements. The last challenge for markerless $\mathrm{AR}$ is the location, which needs to be set up correctly.

None of the presented surveys are systematic, with a research methodology hard to reproduce.

\section{METHODOLOGY}

In this study, a Systematic Literature Review was conducted on usability, mobile AR and cultural heritage. Being systematic, it aims to set up a search protocol, identify all studies that would meet the eligibility criteria and present the findings of the included studies [24]. The study aims to answer the following research questions:

- What kind of usability evaluation did researchers of cultural heritage based mobile augmented reality applications perform?

- How do these types of applications perform? What were the outcomes of the usability evaluation?

The interrogated databases were Web of Science, Scopus, IEEE and Springer. The search was based on the logical expression "mobile augmented reality" AND "cultural heritage" AND ("user experience" OR "usability"). In all four databases, the search was limited to papers published between 2010 and 2020 .

In total, 88 papers were found by searching with the abovementioned keywords. In order to be eligible to be included in this survey, a study needed to meet the following criteria:

- The field of study or the application mentioned in the paper to have as a platform a mobile device (either smartphone or tablet).

- The published language of the study to be English.

- At least one usability or user experience finding regarding MAR used for cultural heritage.

- $\quad$ The study area to be related to culture (here, we took into consideration all of cultural heritage, art, museums, historical sites, and tourism).

Out of the 88 found papers, 57 were excluded due to not meeting one or more eligibility criteria described above, and 31 were kept for further analyzing. Out of the kept researches, 18 of them were found on Springer, 16 on Scopus, 13 on Web of Science and only 2 in IEEE. Some of the studies were found in more than one database.

\section{RESULTS}

All the 31 kept papers have some factors in common, such as the applied domain is cultural heritage, refers to a mobile AR application and has a usability study.

In Table 1 a full comparison of the AR environment and AR types from the surveyed papers is presented. For the environment category, the applicability of the mobile application was considered, as it was created to be used indoors or outdoors. The other category refers to the AR type used - here, the split is made between location-based (when GPS and different sensors are used) and image-based (when QR codes or 2D images are used). Out of the 31 studies, 3 of them $[10,27,33]$ considered indoors use and the AR was based on images. In these cases, the user points the mobile device towards a marker placed in a physical scene and an associated image or painting is displayed on the smartphone's screen. Another study [37] considered indoors use based on location. In this case, a 3D model of a fortified church was virtually displayed on the screen after the physical place was scanned by the sensors first. Another study [13] offer application options for both indoor and outdoor environments based on GPS and sensor tracking. [31] presents different case-studies which employ both indoor and outdoor environments, as well as AR based on location and on images. [7] detects the user's position by using the compass and the accelerometer and when the Point of Interest (POI) is closer than 5 meters, the system uses image-based mode.

Table 1. A full comparison of AR environment and AR types in the surveyed papers

\begin{tabular}{|c|c|c|}
\hline Study & $\begin{array}{l}\text { Environment } \\
\text { (Indoor/Outdoor) }\end{array}$ & $\begin{array}{l}\text { Based on location } \\
\text { (GPS, } \\
\text { sensor)/Based on } \\
\text { images }(\mathrm{QR} / 2 \mathrm{D}) \\
\end{array}$ \\
\hline$[10,27,33]$ & Indoor & Based on images \\
\hline [37] & Indoor & Based on location \\
\hline [13] & Both & Based on location \\
\hline$[7,31]$ & Both & Both \\
\hline $\begin{array}{l}{[1,5,6,8,9,11,1} \\
2,14- \\
20,22,23,25,26 \\
, 28,30,32,34,3 \\
6,38]\end{array}$ & Outdoor & Based on location \\
\hline
\end{tabular}

The other 24 studies relate to mobile applications for outdoors, and all of them employ the location-based AR, which uses GPS and sensors. What can be noticed here is that outdoor applications are prevalent in cultural heritage, and they all are markerless AR, based on location. A reason for that might be, as stated in [13], that enjoyment is higher in the outdoor settings due to the high level of relevance and realism. 
Table 2. Usability testing methods empoloyed in the surveyed papers

\begin{tabular}{|c|c|c|c|c|}
\hline Application & Questionnaire & $\begin{array}{l}\text { Focus } \\
\text { Group }\end{array}$ & $\begin{array}{l}\text { User } \\
\text { Testing }\end{array}$ & Interview \\
\hline 3DGuides platform [22], MobiAR [25] & $\mathrm{x}$ & & & $\mathrm{x}$ \\
\hline $\begin{array}{l}\text { ARAC Maps [10], } 3 \text { interfaces (map, list AR) [12], ArcHIVE } \\
\text { 4Any [19], Flaneur [15], Changdeokgung Palace [20] }\end{array}$ & $\mathrm{x}$ & & $\mathrm{x}$ & \\
\hline $\begin{array}{l}\text { Carpano [33], Mobile AR guide for tourists [34], TowerAR [9], } \\
\text { Virtual/Augmented Gallery [27], Transit Assistant [18], } \\
\text { Finnish Outdoor museum [32], Ai Guang Zhan [8], SitCity } \\
\text { (proposed framework) [36], MixAR [1], "Deoksugung, in my } \\
\text { hands" and DublinAR [16,23], MAR app for Melaka Heritage } \\
\text { Sites [38], OvidAR [6], MAR app to revive a demolished } \\
\text { Reformed Church in Brașov [5], The Historical Tour Guide } \\
\text { [14], 3D model of fortified church [37] }\end{array}$ & $\mathrm{x}$ & & & \\
\hline $\begin{array}{l}\text { MAR application geo-located and gamified [28], } \\
\text { Archaeological park [30], } 2 \text { apps: MTL Urban Museum and } \\
\text { MetaGuide [13], AR workshops [31] }\end{array}$ & & & $\mathrm{x}$ & \\
\hline Open City Museum [17] & & & $\mathrm{x}$ & $\mathrm{x}$ \\
\hline Seraj [7], Millennia Road [26] & $\mathrm{x}$ & & $\mathrm{x}$ & $x$ \\
\hline KnossosAR [11] & $\mathrm{x}$ & $\mathrm{x}$ & & $\mathrm{x}$ \\
\hline
\end{tabular}

In terms of usability, four major testing methods were identified: questionnaires, focus groups, user testing and interviews. Questionnaires were mostly used after the users interacted with the application on-site and frequently included questions targeting the ease of use, the usefulness of the application or the perceived enjoyment. Another method of usability testing which was used only in one study [11] is the focus group. The participants tested the KnossosAR application in four focus groups of four participants each in order to facilitate their live observation by the app developers. Besides the focus group, a questionnaire was also needed to express the overall quality of experience and document any remarks. And finally, a semi-structured interview followed the questionnaire, to offer participants the opportunity to clarify any concerns and suggest additional enhancements. The third usability testing method is represented by user testing. This implies the evaluation of the proposed application in terms of provided functionality and design ease. In user testing, the participants have to perform a set of tasks by using the application and usually the time taken to perform them is measured. In [13], a portable eye-tracking device was also used, as well as audio recordings of verbal interactions between the user and the guide. The last testing method is the interview, implying a face-to-face discussion, either completely informal or semistructured between the users and the observers. In all surveyed articles, the interview was used as a secondary testing method.
In Table 2 a comparison of the usability testing methods is provided. At first glance, it can be noticed that the questionnaire alone was the most used method in the surveyed papers. In most of the studies, the users stated that the application is easy to use and intuitive. They also appreciate the clarity of the interface, the shape and colors of the graphic elements. Several papers $[5,6,11,13,14,16]$ confirmed the enjoyment the users have while using the mobile AR application. Some of those [5,6,11,26,38] also obtained great results regarding the perceived usefulness of the application. [14] even showed that both perceived usefulness and perceived enjoyment had a strong positive effect on the intention of using this type of application. Here, the ease of use can also be a good predictor on the intention to use the app. However, as noted by the authors, tourists have a higher intention in using a mobile AR app for cultural heritage than local residents, who do not feel the same need in their hometown. Likewise, people want to use this type of applications because they enjoy the experience, but also because it helps them achieve some learning objective. Some works as [26], [7] and [11] used three usability testing methods, making their results even more reliable. In all three studies the perceived usefulness, ease of use and enjoyment were confirmed.

In certain studies the usability testing results mention in addition the impact on learning. [34] correlated the ease of use with the ease of learning and [31] suggest that mobile AR 
could be effective in learning processes as a complement to conventional training. In [38], 94\% out of 200 participants preferred the mobile AR application compared to traditional methods of learning. Also, a mobile AR app may increase the users' interest in learning about cultural heritage, as it happened in [37].

A few of the usability downsizes included the high battery consumption for cases in which the AR camera and the GPS were working simultaneously [28]. In [1], the lack of engagement when using the mobile AR app was linked to the low screen resolutions that were set up to ensure a fair exchange between the experience fluidity and the available computational results. Also, the display in mobiles is prone to outdoors light reflections which may cause additional efforts for the visitors. In another study [26], 11 out of 30 participants felt that keeping their heads down to watch the smartphone disconnects them and prevents them from enjoying the surroundings, and five of them found it was "too heavy" to hold the smartphone up while interacting with the guide. In one more study [10] the users said the menu handle was too small - a minor usability flaw which was corrected after the feedback session. Another missing feature was an interactive tutorial when first starting the app [7,34]. Research in [11] showed that users preferred images, audio and narration to textual information, as they claimed it distracted their attention when looking at the POI itself. Furthermore, map environments overcrowded with POIs are restricting the usability [18], as the users are often required to tap on several markers only to be able to locate a particular POI. In the KnossosAR application [11], the authors addressed the occlusion challenge which is usually met in location-based AR applications. They created an efficient method for estimating the field of view of the user, so the POIs would not be obstructed by physical obstacles anymore.

As per gender differences in usability for such mobile applications, [8] found no significant disparities. Nevertheless, [34] found that females rated the experience with AR more satisfying and the interaction better and more intuitive than males.

In [27], 24 out of the 25 study participants expressed their desire to see this type of technology adopted by museums.

\section{DISCUSSION}

The intention of this study was to survey the research in usability testing of mobile AR applications in the cultural heritage field.

There are some limitations to the study that need to be considered. Firstly, only four databases were interrogated, with a specific set of keywords. Future researches could include other relevant databases, such as ACM. Using different keywords might have yielded different results. Considering this, there may be research papers on the subject which are not indexed in the searched databases. Secondly, as a taxonomy for cultural heritage was not found, studies from related fields were included empirically (e.g. tourism, art etc.).

The study hopes to shed a light on the overall user experience of mobile AR applications for cultural heritage. AR has become a great way in helping to preserve, document and explore cultural heritage by bringing people pieces of the past in an interactive and engaging manner. Most of the surveyed papers addressed the usability of their applications via questionnaires. However, only a few studies employed more usability testing methods, especially those which are strictly targeting the interface layout (e.g. eye-tracking devices). Moreover, nearly all the papers referred to outdoor use and location-based AR, as outdoors AR may offer a more natural experience, and being location-based, it offers more accurate results, as sensors and GPS do not rely on lighting conditions.

\section{CONCLUSIONS}

This paper evaluated the research in usability testing of mobile AR applications for cultural heritage. With the aim to be a systematic literature review study, four databases (IEEE, Scopus, Web of Science and Springer) were interrogated by using specific logical expressions. The search found 88 papers, out of which only 31 met the eligibility criteria described in Methodology. All maintained papers have some elements in common, such as the applied domain which is cultural heritage, and they also refer to a mobile AR application and have a usability study.

Findings revealed that $86 \%$ of the papers (without considering the 3 studies which used both types of AR $[7,13,31])$ referred to outdoor use and location-based AR, as outdoors AR may offer a more natural experience, and being location-based, it offers more accurate results. Only a few studies (14\%) targeted the indoors use and were based solely on 2D image recognition.

Moreover, the results showed that most of the applications created were easy to use, intuitive and the study participants enjoyed using them. Also, users appreciate a clear and simple interface. The ease to use the application has a strong impact on the intention to use it. In the same way, the easier to use the mobile AR application is, the easier it is for the user to learn about the content presented. In one study, females rated the experience with AR more satisfying and the interaction better and more intuitive than males. What users did not particularly like were the missing tutorials when first starting the app, the graphic elements too small to interact with, reading a lot of content on a small screen or making efforts to check the screen due to the high brightness conditions of the outdoors, or even having to hold the smartphone up for too long.

What seems to be appreciated in mobile AR apps for cultural heritage are the tutorials from the beginning, the arrows showing how to use the app, the easy to find POIs and a simple, yet intuitive interface. 
The cultural heritage domain can benefit tremendously from the mobile AR applications, as half the population owns a smartphone and it is a great way of preserving, documenting, and exploring all the values it holds. The present survey showed that there is still room for improvement in regard to the user experience of mobile AR applications for cultural heritage. Nevertheless, the field has become quite popular in the past few years, promising an even bigger popularity change.

\section{REFERENCES}

[1] Telmo Adao, Luis Padua, David Narciso, Joaquim Joao Sousa, Luis Agrellos, Emanuel Peres, and Luis Magalhaes. 2019. MixAR: A Multi-Tracking Mixed Reality System to Visualize Virtual Ancient Buildings Aligned Upon Ruins. J. Inf. Technol. Res. 12, 4 (December 2019), 1-33. DOI:https://doi.org/10.4018/JTR.2019100101

[2] Yahaya Ahmad. 2006. The Scope and Definitions of Heritage: From Tangible to Intangible. International Journal of Heritage Studies.

[3] John Aliprantis and George Caridakis. 2019. A Survey of Augmented Reality Applications in Cultural Heritage: International Journal of Computational Methods in Heritage Science 3, 2 (July 2019), 118-147. DOI:https://doi.org/10.4018/IJCMHS.2019070107

[4] Mafkereseb Kassahun Bekele, Roberto Pierdicca, Emanuele Frontoni, Eva Savina Malinverni, and James Gain. 2018. A Survey of Augmented, Virtual, and Mixed Reality for Cultural Heritage. J. Comput. Cult. Herit. 11, 2 (March 2018), 7:1-7:36. DOI:https://doi.org/10.1145/3145534

[5] Razvan Gabriel Boboc, Florin Girbacia, Mihai Duguleana, and Ales Tavcar. 2017. A handheld Augmented Reality to revive a demolished Reformed Church from Brasov. Assoc Computing Machinery, New

York. DOI:https://doi.org/10.1145/3110292.3110311

[6] Rizvan Gabriel Boboc, Mihai Duguleana, GheorgheDaniel Voinea, Cristian-Cezar Postelnicu, DorinMircea Popovici, and Marcello Carrozzino. 2019. Mobile Augmented Reality for Cultural Heritage: Following the Footsteps of Ovid among Different Locations in Europe. Sustainability 11, 4 (February 2019), 1167. DOI:https://doi.org/10.3390/su11041167

[7] Fatiha Bousbahi and Bayan Boreggah. 2018. Mobile Augmented Reality Adaptation through Smartphone Device Based Hybrid Tracking to Support Cultural Heritage Experience. Assoc Computing Machinery, New DOI:https://doi.org/10.1145/3289100.3289109

[8] Chen-Chiou Chiu and Lai-Chung Lee. 2018. System satisfaction survey for the App to integrate search and augmented reality with geographical information technology. Microsyst Technol 24, 1 (January 2018), 319-341. DOI:https://doi.org/10.1007/s00542-0173333-9

[9] M. Duguleana, R. Brodi, F. Girbacia, C. Postelnicu, O. Machidon, and M. Carrozzino. 2016. Time-travelling with mobile augmented reality: A case study on the piazza dei Miracoli. Lecture Notes in Computer Science (including subseries Lecture Notes in Artificial Intelligence and Lecture Notes in Bioinformatics) 10058 LNCS, (2016), 902-912. DOI:https://doi.org/10.1007/978-3-319-48496-9_73

[10] Daniel Eggert, Dennis Hücker, and Volker Paelke. 2014. Augmented Reality Visualization of Archeological Data. In Cartography from Pole to Pole: Selected Contributions to the XXVIth International Conference of the ICA, Dresden 2013, Manfred Buchroithner, Nikolas Prechtel and Dirk Burghardt (eds.). Springer, Berlin, Heidelberg, 203-216. DOI:https://doi.org/10.1007/978-3-642-32618-9_15

[11] P. Galatis, D. Gavalas, V. Kasapakis, G. Pantziou, and C. Zaroliagis. 2016. Mobile augmented reality guides in cultural heritage. DOI:https://doi.org/10.4108/eai.3011-2016.2266954

[12] Dion Hoe-Lian Goh, Chei Sian Lee, and Khasfariyati Razikin. 2011. Comparative Evaluation of Interfaces for Presenting Location-Based Information on Mobile Devices. In Digital Libraries: For Cultural Heritage, Knowledge Dissemination, and Future Creation (Lecture Notes in Computer Science), Springer, Berlin, Heidelberg, 237-246. DOI:https://doi.org/10.1007/9783-642-24826-9_30

[13] Jason M. Harley, Eric G. Poitras, Amanda Jarrell, Melissa C. Duffy, and Susanne P. Lajoie. 2016. Comparing virtual and location-based augmented reality mobile learning: emotions and learning outcomes. Education Tech Research Dev 64, 3 (June 2016), 359-388. DOI:https://doi.org/10.1007/s11423015-9420-7

[14] Anne-Cecilie Haugstvedt and John Krogstie. 2012. Mobile Augmented Reality for Cultural Heritage: A Technology Acceptance Study. In 2012 Ieee International Symposium on Mixed and Augmented Reality (ismar) - Science and Technology. Ieee, New York, 247-255.

[15] Anastasia Ioannidi, Damianos Gavalas, and Vlasios Kasapakis. 2017. Flaneur: Augmented exploration of the architectural urbanscape. In 2017 IEEE Symposium on Computers and Communications (ISCC), IEEE, Heraklion, Greece, 529-533. DOI:https://doi.org/10.1109/ISCC.2017.8024582

[16] Timothy Hyungsoo Jung, Hyunae Lee, Namho Chung, and M. Claudia Tom Dieck. 2018. Cross-cultural differences in adopting mobile augmented reality at cultural heritage tourism sites. Int. J. Contemp. Hosp. 
Manag. $\quad 30, \quad 3 \quad$ (2018), 1621-1645. DOI:https://doi.org/10.1108/IJCHM-02-2017-0084

[17] Georgios Kallergis, Marios Christoulakis, Aimilios Diakakis, Marios Ioannidis, Iasonas Paterakis, Nefeli Manoudaki, Marianthi Liapi, and Konstantinos-Alketas Oungrinis. 2020. Open City Museum: Unveiling the Cultural Heritage of Athens Through an -Augmented Reality Based- Time Leap. In Culture and Computing (Lecture Notes in Computer Science), Springer International Publishing, Cham, 156-171. DOI:https://doi.org/10.1007/978-3-030-50267-6_13

[18] Manousos Kamilakis, Damianos Gavalas, and Christos Zaroliagis. 2016. Mobile User Experience in Augmented Reality vs. Maps Interfaces: A Case Study in Public Transportation. In Augmented Reality, Virtual Reality, and Computer Graphics (Lecture Notes in Computer Science), Springer International Publishing, Cham, 388-396. DOI:https://doi.org/10.1007/978-3319-40621-3 27

[19] Manolya Kavakli. 2015. A people-centric framework for mobile augmented reality systems ( MARS) design: ArcHIVE 4Any. Human-centric Comput. Inf. Sci. 5, (December 2015),

DOI:https://doi.org/10.1186/s13673-015-0055-9

37.

[20] Hayun Kim, Tamas Matuszka, Jea-In Kim, Jungwha Kim, and Woontack Woo. 2017. Ontology-based mobile augmented reality in cultural heritage sites: information modeling and user study. Multimed. Tools Appl. 76, 24 (December 2017), 26001-26029. DOI:https://doi.org/10.1007/s11042-017-4868-6

[21] H. Kolivand, A. El Rhalibi, S. Abdulazeez, and P. Praiwattana. 2018. Cultural Heritage in Marker-Less Augmented Reality: A Survey. In IntechOpen, D. Turcanu-Carutiu and R.-M. Ion (eds.). Intechopen, London. DOI:https://doi.org/10.5772/intechopen.80975

[22] K.I. Kotsopoulos, P. Chourdaki, D. Tsolis, R. Antoniadis, G. Pavlidis, and N. Assimakopoulos. 2019. An authoring platform for developing smart apps which elevate cultural heritage experiences: A system dynamics approach in gamification. Journal of Ambient Intelligence and Humanized Computing (2019). DOI:https://doi.org/10.1007/s12652-019-01505-w

[23] Hyunae Lee, Namho Chung, and Timothy Jung. 2015. Examining the Cultural Differences in Acceptance of Mobile Augmented Reality: Comparison of South Korea and Ireland. In Information and Communication Technologies in Tourism 2015, Springer International Publishing, Cham, 477-491. DOI:https://doi.org/10.1007/978-3-319-14343-9_35

[24] Alessandro Liberati, Douglas G. Altman, Jennifer Tetzlaff, Cynthia Mulrow, Peter C. Gøtzsche, John P. A. Ioannidis, Mike Clarke, P. J. Devereaux, Jos Kleijnen, and David Moher. 2009. The PRISMA Statement for Reporting Systematic Reviews and Meta-
Analyses of Studies That Evaluate Health Care Interventions: Explanation and Elaboration. PLoS Med 6, 7 (July 2009). DOI:https://doi.org/10.1371/journal.pmed.1000100

[25] María Teresa Linaza, David Marimón, Paula Carrasco, Roberto Álvarez, Javier Montesa, Salvador Ramón Aguilar, and Gorka Diez. 2012. Evaluation of Mobile Augmented Reality Applications for Tourism Destinations. In Information and Communication Technologies in Tourism 2012, Springer, Vienna, 260 271. DOI:https://doi.org/10.1007/978-3-7091-1142$0 \_23$

[26] E. Litvak and T. Kuflik. 2020. Enhancing cultural heritage outdoor experience with augmented-reality smart glasses. Personal and Ubiquitous Computing (2020). DOI:https://doi.org/10.1007/s00779-02001366-7

[27] Michele Mallia, Marcello Carrozzino, Chiara Evangelista, and Massimo Bergamasco. 2019. Automatic Creation of a Virtual/Augmented Gallery Based on User Defined Queries on Online Public Repositories. In VR Technologies in Cultural Heritage (Communications in Computer and Information Science), Springer International Publishing, Cham, 135-147. DOI:https://doi.org/10.1007/978-3-03005819-7 11

[28] C. Panou, L. Ragia, D. Dimelli, and K. Mania. 2018. Outdoors mobile augmented reality application visualizing $3 \mathrm{D}$ reconstructed historical monuments. 5967.

[29] C. Perra, E. Grigoriou, A. Liotta, W. Song, C. Usai, and D. Giusto. 2019. Augmented reality for cultural heritage education. $333-336$. DOI:https://doi.org/10.1109/ICCEBerlin47944.2019.8966211

[30] Roberto Pierdicca, Emanuele Frontoni, Primo Zingaretti, Eva Savina Malinverni, Andrea Galli, Ernesto Marcheggiani, and Carlos Smaniotto Costa. 2016. Cyberarchaeology: Improved Way Findings for Archaeological Parks Through Mobile Augmented Reality. In Augmented Reality, Virtual Reality, and Computer Graphics, Pt Ii, L. T. DePaolis and A. Mongelli (eds.). Springer International Publishing Ag, Cham, 172-185. DOI:https://doi.org/10.1007/978-3319-40651-0 14

[31] Ernest Redondo Domínguez, David Fonseca Escudero, Albert Sánchez Riera, and Isidro Navarro Delgado. 2014. Mobile learning in the field of Architecture and Building Construction. A case study analysis. Int J Educ Technol High Educ 11, 1 (January 2014), 152-174. DOI:https://doi.org/10.7238/rusc.v11i1.1844

[32] Kaapo Seppälä, Olli I. Heimo, Timo Korkalainen, Juho Pääkylä, Jussi Latvala, Seppo Helle, Lauri Härkänen, Sami Jokela, Lauri Järvenpää, Frans Saukko, Lauri 
Viinikkala, Tuomas Mäkilä, and Teijo Lehtonen. 2016. Examining User Experience in an Augmented Reality Adventure Game: Case Luostarinmäki Handicrafts Museum. In Technology and Intimacy: Choice or Coercion (IFIP Advances in Information and Communication Technology), Springer International Publishing, Cham, 257-276. DOI:https://doi.org/10.1007/978-3-319-44805-3_21

[33] S. Spacca, E. Dellapiana, and A. Sanna. 2018. Promoting industrial cultural heritage by augmented reality: Application and assessment. Open Cybernetics and Systemics Journal 12, 1 (2018), 61-71. DOI:https://doi.org/10.2174/1874110X01812010061

[34] D. Stoelák, F. Škola, and F. Liarokapis. 2016. Examining user experiences in a mobile augmented reality tourist guide. DOI:https://doi.org/10.1145/2910674.2935835

[35] Ann Marie Sullivan. Cultural Heritage \& New Media: A Future for the Past, 15 J. Marshall Rev. Intell. Prop. L. 604 (2016). NEW MEDIA, 44.

[36] Vanessa Camilleri. 2020. Augmented Reality in Cultural Heritage: Designing for Mobile AR User Experiences. In Rediscovering Heritage Through Technology: A Collection of Innovative Research Case
Studies That Are Reworking The Way We Experience Heritage, Dylan Seychell and Alexiei Dingli (eds.). Springer International Publishing, Cham, 215-237. DOI:https://doi.org/10.1007/978-3-030-36107-5_11

[37] Gheorghe-Daniel Voinea, Florin Girbacia, Cristian Cezar Postelnicu, and Anabela Marto. 2019. Exploring Cultural Heritage Using Augmented Reality Through Google's Project Tango and ARCore. In $\mathrm{Vr}$ Technologies in Cultural Heritage, M. Duguleana, M. Carrozzino, M. Gams and I. Tanea (eds.). Springer International Publishing Ag, Cham, 93-106. DOI:https://doi.org/10.1007/978-3-030-05819-7_8

[38] Syamsul Bahrin Zaibon, Ulka Chandini Pendit, and Juliana Aida Abu Bakar. 2015. Applicability of Mobile Augmented Reality Usage at Melaka Cultural Heritage Sites. In Proceedings of the 5th International Conference on Computing \& Informatics, Z. Jamaludin, N. ChePa, W. H. W. Ishak and S. B. Zaibon (eds.). Univ Utari Malaysia-Uum, Sintok, 235-240.

[39] Smartphone users worldwide 2020. Statista. Retrieved July 21, $2020 \quad$ from https://www.statista.com/statistics/330695/number-ofsmartphone-users-worldwide/ 